\title{
Una alternativa progresiva a la estructura lineal de precios públicos en la financiación del sistema universitario en España*
}

\author{
MARÍA GIL IZQUIERDO ${ }^{\text {a }}$, FABIO CARTA ${ }^{b}$ \\ a Universidad Autónoma de Madrid, Facultad de Ciencias Económicas y Empresariales, Campus de \\ Cantoblanco, Avda. Francisco Tomás y Valiente, 5, 28049 Cantoblanco (Madrid), España. E- \\ mail: maria.gil@uam.es \\ ${ }^{b}$ Universidad de Alcalá de Henares, Escuela de Doctorado, c/ Libreros, 21, 28801 Alcalá de \\ Henares, Madrid, España.E-mail: fabio.carta@edu.uah.es
}

\begin{abstract}
RESUMEN
Este trabajo analiza los principales cambios que se han introducido recientemente en la financiación del Sistema Universitario Español. El objetivo es, por una parte, revisar las consecuencias derivadas de estas medidas en términos de suficiencia y equidad y, por otra, estudiar la viabilidad de aplicar un sistema progresivo en el cobro de precios públicos, teniendo en cuenta el origen económico del estudiante. Las conclusiones evidencian que la introducción de un sistema de este tipo sería compatible con los principios de suficiencia y de equidad, y viable en su aplicación. Sin embargo, se requieren algunas precauciones para que los efectos en recaudación, progresividad y redistribución tengan el resultado perseguido.
\end{abstract}

Palabras clave: Progresividad, precios de matrícula, fondos públicos, becas, universidad.

\section{A Progressive Alternative to the Linear Structure of Tuition Fees in the Spanish Higher Education Financing System}

\begin{abstract}
This paper analyzes the substantial changes that have been introduced recently in the Spanish University System funding. The aims are, first, to review the consequences of these measures in terms of adequacy and equity and, secondly, to provide an accurate discussion of the feasibility of applying a progressive system in public prices, taking into account the student's economic background. The findings show that the introduction of such a system would be compatible with the principles of adequacy and equity, so as viable in its application. However, some design precautions are also highlighted, so that the effects on revenue, progressivity and redistribution are the desirable ones.
\end{abstract}

Keywords: Progressivity, Tuition Fees, Public Funds, Scholarships, Higher Education.

Clasificación JEL: I22, I24

\footnotetext{
* Los autores agradecen los comentarios y sugerencias recibidos de Oriol Escardibul, así como de dos evauladores anómimos, cuyas aportaciones han contribuido a mejorar la versión última de este articulo.
}

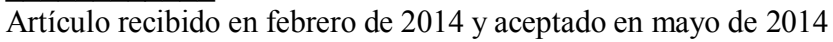

Artículo disponible en versión electrónica en la página www.revista-eea.net, ref. ə-32306 


\section{INTRODUCCIÓN}

El Real Decreto-ley 14/2012 (BOE, 2012a), de medidas urgentes de racionalización del gasto público en el ámbito educativo, ha introducido un importante incremento en los precios públicos pagados por los estudiantes universitarios. Esta decisión tiene su origen tanto en la llamada Estrategia Universidad 2015 como en una serie de medidas que encuentran su justificación política en la coyuntura económica actual.

Precisamente la situación de crisis económica ha dificultado aún más la aceptación de esta decisión por parte de diferentes sectores de la sociedad, ya contrarios a un aumento del gasto privado en la educación pública superior, más aún en un contexto de subida de impuestos y de recortes en otros gastos públicos. De hecho, una de las principales justificaciones en contra de la subida de los precios públicos universitarios es que unos precios elevados pueden suponer una importante barrera de entrada o de permanencia para los estudiantes con menos recursos.

Para compensar las posibles desigualdades debidas a la renta de origen, España pertenece al modelo, dentro los países de la OCDE, que descansa fundamentalmente en un sistema de becas. Sin embargo, debido a los recortes presupuestarios, ciertos aspectos de este sistema han sido recientemente modificados; esto, unido a la subida de precios, puede comprometer la consecución real de la igualdad de oportunidades en el Sistema Universitario Español (SUE). En cualquier caso, no hay que olvidar que el actual sistema de cobro de precios públicos es regresivo en sí mismo ${ }^{1}$ : todos los estudiantes pagan los mismos precios independientemente de la renta de su hogar de origen, debiéndose la diferenciación en los precios al consumo de convocatorias, a la experimentalidad de la rama elegida y a la comunidad autónoma en que se sitúe la universidad.

El objetivo fundamental de este trabajo es el de evidenciar la existencia de soluciones alternativas a la estructura lineal de precios públicos del SUE, que, si bien diseñadas, permitirían conciliar la necesidad de recursos suficientes e inmediatos con la necesidad de garantizar el acceso a cualquier estudiante independientemente de su situación económica, en particular -aunque no sólo- en un contexto de crisis económica como el actual. Dicho objetivo surge de la constatación de los límites de los sistemas de financiación universitaria existentes evidenciados por la literatura, de las características socioeconómicas

\footnotetext{
${ }^{1}$ Más aún, la subvención generalizada a todos los estudiantes independientemente de su rendimiento académico o nivel de renta no provoca mejoras en la eficiencia interna, ni en la suficiencia, ni en la equidad institucional o en la igualdad de oportunidades en el acceso (Hernández y Pérez, 2011).
} 
españolas y de la escasa atención que un sistema de este tipo ha recibido en el ámbito de los estudios de economía de la educación.

Por ello, la organización del trabajo es la siguiente. En un primer apartado, se revisa el papel que juegan los precios públicos en la consecución de los principios de suficiencia y equidad de los sistemas de financiación y cuál es la posición de España respecto a otros países de su entorno. A continuación, y en dos apartados diferenciados, se estudian los importantes cambios que en fechas recientes se han producido tanto en la política de precios públicos como en la de becas, teniendo en cuenta el marco teórico anteriormente descrito y mostrando cómo pueden haber afectado al principio de equidad. La principal aportación de este trabajo es la de explorar la posibilidad de introducir un sistema de financiación alternativo que pueda paliar los posibles efectos negativos del incremento de precios públicos, no sólo a través del sistema de becas, sino interviniendo en la progresividad de dichos precios. Para ello se dedica un epígrafe a la descripción y valoración de dos sistemas que aplican criterios de progresividad en el cobro de sus precios universitarios: Italia y Cataluña. Por último, y antes de las conclusiones, se propone una sección de análisis y valoración de la viabilidad de la aplicación de esta medida en el contexto actual del SUE.

\section{ARGUMENTOS DE TEORÍA ECONÓMICA Y COMPARATIVA INTERNACIONAL}

En general, cualquier sistema de financiación universitario debe cumplir los objetivos fundamentales de suficiencia, eficiencia y equidad. Esto es, las universidades públicas deben tener recursos suficientes para poder cumplir con sus funciones, tienen que maximizar sus resultados a un determinado coste y, por último, tienen la tarea de garantizar la igualdad de oportunidades en el acceso y en la permanencia de sus alumnos (San Segundo, 2005; Salas, 2008). La conciliación de estos objetivos no es una tarea fácil y depende de múltiples factores (políticos, económicos y sociales). Por otro lado, del análisis de los modelos de financiación de la educación superior vigentes en la actualidad (véase OCDE, 2013), se desprende que existen formas muy diferentes de entender y de repartir la financiación de este nivel educativo entre fuentes públicas y privadas.

Centrándonos en la financiación privada a cargo de los usuarios de educación superior, cargar con parte del coste de este servicio a sus beneficiarios es algo común a todos los sistemas de financiación de los países de la OCDE (con excepción de los países nórdicos ${ }^{2}$, que abogan por la gratuidad de las matrículas

\footnotetext{
${ }^{2}$ Éstos son los únicos países en los cuales en ningún caso se cobran precios públicos. Existe otro grupo de países (Croacia, Estonia, Polonia y Rusia), en los que existe un sistema de precios dual que determina qué parte del alumnado no tiene que pagar dichos precios de matrícula. Sin embargo, en la mayoría de estos últimos países, cerca del 50\% de los estudiantes deben pagar por sus matrículas. También hay que señalar el caso de Grecia, donde los estudiantes pagan sólo en
} 
universitarias). Sin embargo, existe un amplio abanico de posibilidades al determinar el porcentaje de la contribución requerida al estudiante: de países que cobran matrículas moderadas o reducidas -que rondan entre el $10 \%$ y el $20 \%$ del coste total del servicio (México y la mayoría de países de la UE)- a los que marcan unos precios elevados -que superan los 1.500 dólares (países anglosajones)- o muy elevados -que cubren casi totalmente el coste de la prestación del servicio (Corea o Japón)-.

Todos estos modelos de financiación ponen en duda el principio de equidad ${ }^{3}$ $\mathrm{y}$, en algunos casos, pueden comprometer el de suficiencia. Si los precios cobrados a los estudiantes son iguales independientemente de la renta de origen, ya desde el diseño de esta parte de la financiación se incumple con el argumento de equidad vertical ${ }^{4}$. En el caso de la suficiencia, precios públicos reducidos (modelo en que se encuadra España) pueden impedir la provisión de recursos necesaria para garantizar un sistema educativo de calidad, si el sector público no puede asumir el coste total (o una parte significativa del mismo) de prestar este servicio.

Este último motivo ha justificado que en las últimas décadas la financiación vía precios públicos haya adquirido una mayor relevancia y que la mayoría de los países de la OCDE haya tomado la decisión de aumentar el importe de dichos precios. Además, en los últimos años se han añadido otros aspectos que han reafirmado esta decisión: la posibilidad de garantizar, gracias a los precios públicos, recursos inmediatos; la de generar una relación más directa entre el estudiante y la universidad, aumentando la exigencia de calidad por parte del primero y la rendición de cuentas de la segunda; y la de exigir un mayor aprovechamiento de los recursos públicos a quienes se benefician de ellos.

\section{EVOLUCIÓN RECIENTE Y ESTADO ACTUAL DEL SISTEMA DE PRECIOS PÚBLICOS POR MATRICULACIÓN EN ESPAÑA}

España se encuadra en el modelo de financiación caracterizado por cobrar precios públicos reducidos, además de ser uno de los pocos países de la OCDE que no ha modificado su sistema de precios públicos hasta 2012.

\footnotetext{
cursos de posgrado específicos y en la Universidad Abierta de Grecia, y el caso de Escocia, en el que se cobra a los estudiantes de posgrado, de tiempo parcial y a los que estudian para obtener un segundo grado (OCDE, 2008).

${ }^{3}$ Tampoco la gratuidad total es totalmente equitativa, ya que los individuos que no siguen sus estudios superiores y que tienen un menor nivel de renta subsidiarían la educación de aquellos con mayores recursos económicos. Además, puede generar un exceso de capital humano excesivamente cualificado, con las pérdidas de eficiencia derivadas de ello (García-Peñalosa y Wälde, 2000).

${ }^{4}$ Entendida como que quien más recursos tiene, más debe aportar.
} 
Hasta esa fecha, cada comunidad autónoma establecía el precio que consideraba oportuno, en función de los criterios de experimentalidad de la enseñanza y de utilización e intensificación del servicio universitario (repetición de asignatura), pero siempre dentro de una horquilla, aprobada anualmente por la Conferencia General de Política Universitaria (CGPU), constituida por el Ministerio de Educación y todas las CC. AA. Una vez determinado el precio máximo y el mínimo, cada comunidad autónoma, a través de sus correspondientes órganos (por ejemplo, los Consejos Interuniversitarios) decidía en qué punto de ese intervalo iba a situar los precios de matrícula que regirían los estudios oficiales de las universidades bajo su competencia. El precio promedio nacional se situaba ligeramente por encima de los $1.000 €$ anuales, aunque este precio no reflejaría el importe al que se enfrenta un alumno que se matricula por primera vez de un curso o que consigue aprobar un curso al año, sino que promedia las posibles repeticiones de asignaturas, con el consiguiente aumento en los precios públicos pagados. En 2011, la media para todas las CC. AA. indicaba que el precio medio de la segunda matrícula ${ }^{5}$ era un $34,4 \%$ superior al de la primera, y el de la tercera un $87,9 \%$ superior a la primera (MECD, 2011).

En 2012 se aprueba el Real Decreto-Ley 14/2012, de 20 de abril, de medidas urgentes de racionalización del gasto público en el ámbito educativo. Entre otras, se proponen medidas de actuación que se refieren a la política de precios públicos universitarios, que recogen muchas de las recomendaciones del Documento de 2010 del Consejo de Universidades ${ }^{6}$. Sin suponer un cambio drástico con respecto al sistema anterior en relación al papel de los actores involucrados y a la estructura general (se fija la horquilla de precios por la CGPU, sigue siendo lineal en el cobro según renta y diferenciado según experimentalidad, repetición y C. A.), se propugna un aumento importante de los precios pagados por los estudiantes, sobre todo a medida que aumenta el número de repeticiones. Esta medida se aplica desde el curso 2012/13, y se fija el curso 2019/20 como horizonte temporal de dichos incrementos. Además, permite establecer precios diferenciados para cada estudio en función del coste real del servicio ${ }^{7}$ y del tipo de matrícula, previo acuerdo de la CGPU. En concreto, «los precios públicos cubrirán entre el $15 \%$ y el $25 \%$ de los costes en primera matrícula; entre el $30 \%$ y el $40 \%$ de los costes en segunda matrícula; entre el $65 \%$ y el $75 \%$ de los cos-

\footnotetext{
${ }^{5}$ Desde la introducción del Espacio Europeo de Educación Superior (EEES) las repeticiones de asignaturas conllevan segundas y terceras matriculaciones, permitiendo en general un número más reducido de oportunidades de volver a examinarse respecto a las antiguas convocatorias de asignaturas en las licenciaturas.

${ }^{6}$ Véase Albert y Roig (2013) para una revisión detallada de los cambios que ha supuesto este decreto en términos de precios públicos durante el curso 2012/2013.

${ }^{7}$ Esto implica la necesidad de implantar un sistema de contabilidad analítica en las universidades que permita determinar dichos costes reales.
} 
tes en la tercera matrícula; y entre el $90 \%$ y el $100 \%$ de los costes a partir de la cuarta matrícula».

La concreción de esta medida ha supuesto que, en términos generales, todas las CC. AA. hayan incrementado los precios, sobre todo en las segundas matrículas, y especialmente en las terceras y cuartas desde el curso 2011/12. En 2012/13, el precio medio del crédito que se matricula por primera vez aumenta en un $16,7 \%$ de media de respecto al curso anterior, la mayor subida desde que se aprobaron los Grados (los incrementos fueron del 5\% y 6\% durante los cursos previos, respectivamente, y del 3\% en el curso 2013/14 respecto a 2012/13). Por lo que respecta a segundas y siguientes matrículas, en la Tabla 1 se aprecia la penalización a la repetición de matrícula, creciente con el número de matriculaciones, de tal forma que matricularse por cuarta vez supone, en media, 70 euros más que hacerlo por primera vez.

Tabla 1

Precios medio (euros) por crédito matriculado, varias matrículas, cursos $2013 / 14$ y 2012/13

\begin{tabular}{|c|c|c|}
\hline & $\mathbf{2 0 1 3 / 1 4}$ & $\mathbf{2 0 1 2 / 1 3}$ \\
\hline $\mathbf{1}^{\mathbf{a}}$ matrícula & 18,37 & 18,28 \\
\hline $\mathbf{2}^{\mathbf{a}}$ matrícula & 30,49 & 30,70 \\
\hline 3 $^{\mathbf{a}}$ matrícula & 63,15 & 63,58 \\
\hline $\mathbf{4}^{\mathbf{a}}$ matrícula & 89,18 & 89,70 \\
\hline
\end{tabular}

Fuente: Elaboración propia a partir de Estadística de Precios Públicos Universitarios, varios años (Ministerio de Educación).

Por CC. AA., la concreción del RDL 14-2012 ha sido muy desigual. Así, los precios medios por crédito (con diferencias según experimentalidad) de primeras matrículas son en general inferiores a $20 €$ (en algunas CC. AA., mucho menores, como es el caso de Andalucía, Cantabria o Galicia, que rondan los $13 €$, y en otras, mucho mayores, como es el caso de Madrid o Cataluña, con precios superiores a los $30 €$ ). Las segundas matriculaciones rondan los $30 € /$ crédito, mientras que las terceras y cuartas superan en muchos casos los $60 €$ y $100 €$, respectivamente (MECD, 2014). En definitiva, el mayor de los cambios que introduce el R. D. es que se refuerza claramente la penalización por repetición de asignatura (medida aplicada en todas las CC. AA. a partir de 2012/13, Figura1) y se incrementa el precio pagado por las primeras matrículas, pasando de unos $879 €$ anuales promedio (considerando un curso de 60 créditos) en 2010/11 a unos $1.105 €$ anuales en 2012/13, si bien existe una gran dispersión atendiendo a la Comunidad Autónoma donde se sitúe la universidad y a la experimentalidad elegida. 
Figura 1

Incrementos en $3^{\mathrm{a}}$ o $4^{\mathrm{a}}$ matrículas y sucesivas, por CC. AA.

Cursos 2010/11, 2012/13 y 2013/14

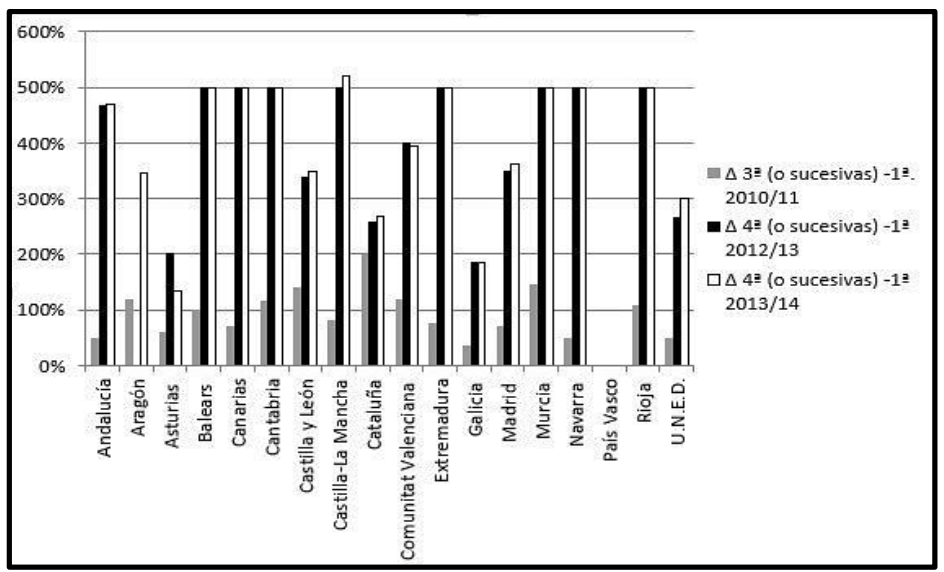

Fuente: Elaboración propia a partir de Estadística de Precios Públicos Universitarios, varios años (Ministerio de Educación).

\section{EVOLUCIÓN RECIENTE Y ESTADO ACTUAL DEL SISTEMA DE BECAS}

Un sistema de precios públicos elevados puede generar efectos negativos, ya que puede suponer para determinados colectivos una barrera de entrada o de continuidad de los estudios universitarios. Por ello, todos los modelos de financiación cuentan, en mayor o menor medida, con sistemas de ayudas a los estudiantes. La configuración de estas ayudas en forma, principalmente, de préstamos o de becas, su cuantía y el número de estudiantes a los que cubre, caracterizan a cada uno de dichos modelos. España se sitúa en el grupo de países que dirige sus esfuerzos hacia una fuerte protección a los estudiantes de hogares con rentas más bajas; por el contrario, los préstamos renta tienen aún un peso escaso en la financiación a estudiantes en nuestro país ${ }^{8}$. España es uno de los países de la OCDE que destina un porcentaje más bajo de su PIB a la inversión pública en becas y ayudas en educación superior -media de la OCDE: $0,31 \%$ vs. el $0,11 \%$ de España, en 2010-, muy alejado de los valores de otros países, como los nórdicos o los anglosajones. En relación al gasto público en educación superior,

\footnotetext{
${ }^{8}$ No se abordará en este apartado el análisis de los préstamos renta como instrumento de financiación a estudiantes, ya que excede el objetivo de este trabajo. Sí se puede señalar, a modo de resumen, que los préstamos representan un instrumento de financiación accesible para un mayor número de estudiantes en la medida que se les transfiere parte de los costes de la educación superior (Martín, 2009), con buenas propiedades en términos de eficiencia, de calidad y de equidad (Salas, 2008), si bien en España no están tan generalizados como en otros países de nuestro entorno ( $0.6 \%$ de inversión pública en préstamos-renta frente al $7.8 \%$ de la media de la OCDE).
} 
España dedica el 9,2\% a ayudas directas a estudiantes, mientras que la media de la OCDE es de 11,4\% (OCDE, 2013).

En los últimos años, se han producido ciertos cambios importantes en el sistema general de becas, tanto en la evolución de las cuantías destinadas a este epígrafe de gasto y en el número de becarios, como en las modalidades y requisitos de las becas, que alcanzarán su culminación en 2020 (Estrategia 2015). Desde la convocatoria del curso 2010/11 se recogen diferentes modalidades de becas convocadas por la Administración General del Estado, cuya concesión se establece en función de diferentes requisitos económicos, académicos y de discapacidad. Por una parte, las destinadas a garantizar la igualdad de oportunidades y que constituyen el grueso del presupuesto, que son, fundamentalmente, las becas salario y compensatorias (sustitutivas de un salario, ascienden a $3.500 €$ y las reciben un $3,19 \%$ de los estudiantes de grado) y las de movilidad (que pretenden evitar que la elección del centro se vea motivada por la renta familiar o la oferta académica de proximidad); por otra, las de movilidad internacional, las de excelencia académica, y otras modalidades (como la exención de pago de precios públicos).

En términos presupuestarios, la inversión española en becas creció entre 2004 y 2009 en un $175 \%$, por encima del incremento experimentado en media en la OCDE $(107,1 \%)$, si bien a partir del año 2011 se han producido reducciones en las cantidades de gasto recogidas en los Presupuestos Generales del Estado en este concepto: de un 2,89\% en 2013 respecto al año anterior, valor que asciende al 9,83\%, si se comparan los Presupuestos de 2013 y 2011 (Ministerio de Hacienda y Administraciones Públicas, varios años). A pesar de estos decrementos, y si nos centramos en las becas generales (convocatoria general y de movilidad), los datos (MECD, 2013a) muestran que el número de becarios ha crecido un $14,2 \%$ en el curso $2011 / 12$ respecto al año anterior y un $56,6 \%$ respecto al curso 2004/05. También han aumentado sensiblemente las cantidades dedicadas a este tipo de becas. Para el último año del que se dispone de información (MECD, 2013, con datos referidos al curso 2011/12), se puede constatar que el importe total ha crecido un $18,1 \%$ entre $2011 / 10$ y $2011 / 12$, un $97,1 \%$ entre los cursos 2011/12 y 2004/05. Finalmente, hay que señalar que desde el año 2007 todos los becarios que cumplen los requisitos reciben la beca que merecen, cumpliéndose así el que las becas constituyan un derecho del estudiante y no una convocatoria competitiva.

Las modificaciones en las modalidades y las cuantías de las becas, unidas a las restricciones presupuestarias, han desembocado en una mayor presión tanto para los hogares -los requisitos son más estrictos- como para el Estado, ya que aumenta la población que potencialmente tiene derecho a recibir una beca general (los umbrales de renta para tener derecho a una beca se mantienen en las 
últimas convocatorias a la vez que la renta media por hogar decrece9 $)$. Además, como los presupuestos reducen el montante total dedicado al epígrafe de becas y ayudas a familias e instituciones sin fines de lucro, es de esperar que los conceptos que se resientan sean los dedicados a la movilidad de internacional o inter-universidades (la aportación del Estado a las becas Erasmus y Séneca se ha reducido en un $70 \%$ entre 2011 y 2013). La implicación de este esquema supone, desde el punto de vista económico, priorizar la igualdad de oportunidades en el acceso a la universidad a costa de los beneficios asociados a la movilidad de los universitarios ${ }^{10}$.

Otra cuestión es la referida a los requisitos académicos. La convocatoria para el curso 2013/14 (R.D. 1000/2012, BOE, 2012b) plantea endurecer en gran medida el porcentaje de créditos que los becarios han de superar para tener acceso a las becas o la nota media que se debe conseguir en las asignaturas superadas. Además, se renuncia a financiar terceras o cuartas y sucesivas matrículas dependiendo de la rama de estudios cursada. Por otra parte, hasta el curso 2012/13, si no se alcanzaba el porcentaje de aprobados, el becario no tenía que devolver la prestación, sino que se le denegaba su solicitud de beca al año siguiente. Sin embargo, a partir de dicho curso, los becarios deberán devolver el importe recibido de la beca (a excepción de la exención de precios), si no han superado el $50 \%$ de los créditos matriculados. Todo ello supone elevar enormemente la exigencia académica a los becarios, muy por encima de la que se exige al resto de estudiantes ${ }^{11}$.

A esta presión por mantener la beca se une el problema relativo a la lentitud en el cobro de las ayudas concedidas, que suele producirse habitualmente bien entrado el curso académico. Los inconvenientes asociados a esta tardanza pueden convertirse en decisivos en momentos como el actual en los que son habituales las situaciones sobrevenidas de desempleo o de reducciones de ingresos en los hogares de los estudiantes, lo que puede desembocar en un abandono de los estudios universitarios por una parte importante del colectivo de becarios, con el consiguiente perjuicio de la igualdad de oportunidades. En este sentido, muchas universidades ${ }^{12}$ han creado o reforzado sus fondos propios dedicados a "rescatar" a determinados estudiantes que se verían forzados a abandonar sus estudios en algunas de las situaciones antes señaladas.

${ }^{9}$ Con los últimos datos disponibles, un 19.2\% entre 2008 y 2011 (INE, 2013).

${ }^{10}$ No existen requisitos económicos para la concesión de las becas Erasmus y Séneca.

${ }^{11} \mathrm{Si}$ bien es cierto que en los últimos años se han endurecido las normas de permanencia (reduciendo el número de matriculaciones posibles) y se penaliza claramente a los repetidores a través del sistema de precios públicos.

${ }^{12}$ Como la universidad de Burgos, la Autónoma de Madrid, la Autónoma de Barcelona, o la UNED, entre otras. 


\section{UN SISTEMA PROGRESIVO EN EL COBRO DE PRECIOS PÚBLICOS: EL CASO ITALIANO Y EL CATALÁN}

La regresividad de los precios públicos y las debilidades del sistema de becas, así como el contexto socioeconómico actual, hacen necesaria la búsqueda de unos mecanismos de financiación de la educación superior que, de forma compatible con la estructura socioeconómica de cada país y más allá del contexto de crisis, generen recursos suficientes e inmediatos, de manera eficiente y bajo un esquema equitativo respecto a la contribución de cada estudiante.

El sistema de financiación italiano reúne interesantes características en el ámbito de nuestro estudio, ya que su estructura de precios públicos se articula a través de un sistema progresivo, que tiene en cuenta la condición económica del estudiante. El Gobierno establece a nivel nacional las normas básicas relativas a la llamada contribución estudiantil (DPR 306/1997, GU, 1997), que, por analogía con el caso español, sería el equivalente de los precios públicos universitarios. Ésta incluye dos conceptos: la tasa de matriculación, cuyo importe mínimo establece el Ministerio de Instrucción, Universidad e Investigación (MIUR, en su sigla italiana), y las contribuciones universitarias, determinadas autónomamente por las universidades, según «criterios de solidaridad y equidad, en relación a las condiciones económicas del inscrito».

La estimación de la condición económica del estudiante sigue principalmente los criterios establecidos en el decreto legislativo 109/1998 (GU, 1998), válidos para solicitar cualquier prestación pública social. En términos prácticos, se presenta en los centros autorizados la Declaración Sustitutiva Única (DSU), que contiene las informaciones necesarias para poder determinar el Indicador de la Situación Económica Equivalente (ISEE), que tiene validez anual. Éste se calcula a partir de la suma de las rentas y del $20 \%$ del patrimonio (inmobiliario y financiero) de los miembros del hogar. Además, se le aplica una escala de equivalencia, que tiene en cuenta el número de integrantes y ciertos casos particulares de la situación familiar. Las universidades y los demás entes públicos que ofrecen prestaciones sociales pueden establecer otros criterios para integrar el cálculo del ISEE.

Teniendo en cuenta lo anterior, cada universidad establece sus precios públicos y los distribuye autónomamente en diferentes tramos en función de la posición económica del hogar determinada a través del ISEE/ISEEU ${ }^{13}$. Por tanto, el número, el rango y el importe pagado en cada uno de los tramos varían en base a la política de contribución estudiantil de cada universidad. El estudiante sabe, mediante su certificación ISEE/ISEEU, el tramo de renta en el que se sitúa y, por lo tanto, el importe de la contribución que deberá pagar, importe que se

${ }^{13}$ Las universidades actualizan y publican anualmente dichos valores, junto con las normas de matriculación. 
incrementa a medida que lo hace la renta del hogar al que pertenece. Además, a las universidades corresponde el establecimiento de criterios para la exención total o parcial del pago de las contribuciones para los estudiantes con alto rendimiento académico o sin recursos. Igualmente, están obligadas a incluir entre los estudiantes exentos del pago los beneficiarios de becas o préstamos de honor ${ }^{14}$.

Sin profundizar en los diferentes importes de contribución estudiantil que fijan las universidades italianas, resulta evidente que una estructura de este tipo conlleva una extraordinaria heterogeneidad en el sistema de precios públicos. Sin embargo, lo que aquí se pretende subrayar es que el establecimiento de la contribución estudiantil se realiza en base a la situación económica de cada hogar. En otros términos, en el sistema de financiación vía precios públicos se introducen mecanismos de progresividad, que modifican las condiciones de acceso de los estudiantes y que afectan al principio de equidad, tal y como se aprecia en la Figura 2, en el que se comprueban los mayores precios pagados por matrícula a medida que el estudiante se sitúa en un escalón superior de renta.

Figura 2

Importe medio de los precios públicos en Italia por tramos de renta, 2011

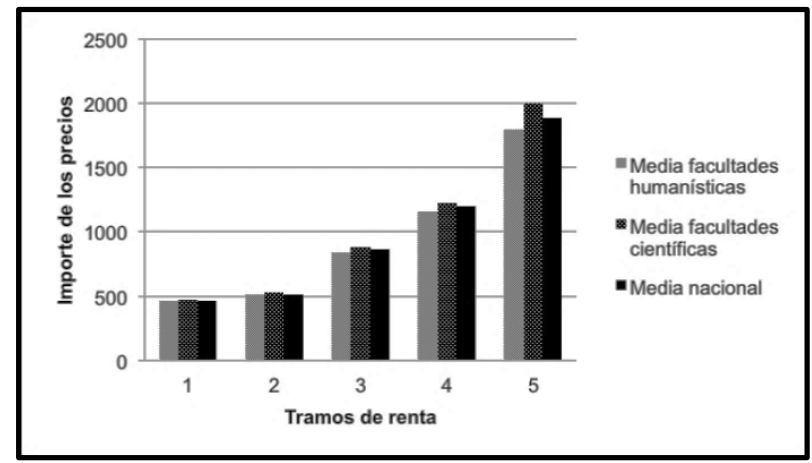

Fuente: Elaboración propia a partir de Federconsumatori (2011).

En el año académico 2012/2013, Cataluña introdujo un sistema relativamente similar al italiano. De hecho, si bien la estructura final de los precios públicos se configura de manera progresiva, los mecanismos para alcanzar dicha estructura y el papel de las universidades son distintos. Ello es también

${ }^{14}$ Dichos préstamos, basados en el honor del estudiante (que no tienen que ver con las Matrículas de Honor en España), se establecen a partir de convenios entre universidades y bancos, y se conceden con condiciones financieras particularmente favorables. 
consecuencia de la potestad de la Generalitat de Catalunya, que limita su actuación en materia educativa dentro del ámbito de su competencia autonómica.

Así, en una fase inicial, los precios públicos catalanes reflejan el marco normativo estatal: se mantiene su estructura lineal, aumenta la contribución del estudiante al coste total en las sucesivas matriculaciones ${ }^{15}$ y se reproduce una diferenciación de los precios de los créditos en función de la experimentalidad del estudio, mediante una clasificación denominada coeficiente de estructura docente ${ }^{16}$. Sin embargo, a través de las llamadas becas Equitat, la Generalitat introduce en el sistema de precios públicos un mecanismo de aportación progresiva de los estudiantes. Estas "becas", a pesar de su nombre, no constituyen una transferencia monetaria hacia los estudiantes, ni otorgan el derecho a utilizar determinados servicios a título gratuito, como comedores o residencias, sino que conceden, a los estudiantes que la solicitan, una reducción de los precios públicos en base al nivel de renta de su hogar (art. 4, Decret 77/2012).

La convocatoria de las becas Equitat establece seis tramos de renta diferentes, que varían en base al número de miembros de la unidad familiar ${ }^{17}$. En el caso, por ejemplo, de un hogar formado por 4 miembros, los límites máximos de renta de cada tramo son respectivamente de $38.831 €$ para el primero, 39.733 $€$ para el segundo, $40.635 €$ para el tercero, $41.536 €$ para el cuarto, $45.144 €$ para el quinto y al último tramo corresponden todas las rentas superiores ${ }^{18}$. Además, se establece un tramo inicial, denominado tramo 0 , que incluye exclusivamente los beneficiarios de la beca MECD, los cuales reciben de forma complementaria una beca Equitat al fin de quedar completamente exentos del pago de la matrícula, mientras que al tramo 1 corresponden los estudiantes que cumplen los requisitos económicos establecidos por el MECD, pero no los académicos. En base al tramo en el que se sitúe el estudiante y al coeficiente de estructura docente de los estudios elegidos, varía el importe final de los precios públicos que paga el estudiante, a partir de una minoración porcentual en el precio del crédito matriculado por primera vez, respectivamente del $50 \%, 40 \%, 30 \%, 20 \%$, $10 \%$ y $0 \%$. El estudiante, en términos prácticos, procede al pago de la matrícula

${ }^{15}$ La contribución del estudiante al coste total aumenta en un 30\%, 65\% y $90 \%$, respectivamente para segundas, terceras y posteriores matrículas. Estos valores equivalen a los porcentajes mínimos establecidos en el Real Decreto-ley 14/2012.

${ }^{16}$ Los precios de los créditos se distribuyen en tres niveles de estructura docente: A (ciencias sociales, en su mayoría), B (ciencias naturales e ingenierías), C (ciencias médicas y de la salud).

${ }^{17}$ El cálculo de la renta familiar se basa en la normativa relativa al Impuesto sobre la Renta de las Personas Físicas (IRPF). Además, la suma de los valores catastrales de la unidad familiar, con la exclusión de la vivienda habitual, no puede superar el importe de $42.900 €$.

${ }^{18}$ En la última convocatoria 2013/2014 los valores han sido los siguientes: $38.831 €$ para el primer tramo, 41.719,65€ para el segundo, 42.666,75€ para el tercero, 43.612,80€ para el cuarto y 47.401,20 € para el quinto (AGAUR, 2013b). 
en dos fases: una primera, en el momento de su formalización, en la que se le exige el $60 \%$ del precio público del crédito; una segunda, una vez resuelta la convocatoria, en la que el estudiante abona hasta el $40 \%$ restante (el $30 \%$ en la convocatoria de 2013/14) y, en el caso de haber aportado más de lo debido, recibe la devolución del importe en exceso.

Este sistema conduce a una diferente contribución final de los estudiantes al coste total dependiendo de su situación económica. La Tabla 2 muestra el porcentaje de aportación en base al tramo de renta familiar al que pertenece el estudiante, pasando del $0 \%$ (los becarios del MECD) hasta el $25 \%$ para las rentas superiores a $45.144 €$, si se considera un hogar formado por 4 miembros. Además, en la tabla también se indica el incremento porcentual en el importe de los precios públicos que se registra en cada tramo respecto al año académico anterior $^{19}$. Los primeros tres tramos no experimentan aumentos y, según la estimación de la Generalitat, equivalen al 30\% de los estudiantes. Por su parte, en los tres tramos más ricos se observa un importante incremento de los precios públicos, respectivamente del $33,3 \%, 50 \%$ y hasta el $66,7 \%$. Estos aumentos corresponden al $65 \%$ de los estudiantes y afectan a aquéllos cuya renta familiar anual es superior a $\operatorname{los} 41.536 €$, manteniendo el ejemplo de una unidad familiar formada por 4 miembros.

\section{Tabla 2}

Aportación del estudiante al coste total por tramos de renta e incremento del precio público

\begin{tabular}{cccc}
\hline Tramo de renta & $\begin{array}{c}\text { \% aportación } \\
\text { estudiante al } \\
\text { coste total }\end{array}$ & $\begin{array}{c}\text { \% incremento } \\
\text { precio } \\
\text { público }\end{array}$ & $\begin{array}{c}\% \\
\text { estudiantes* }\end{array}$ \\
Tramo 0 & 0 & (exención) & 15 \\
Tramo 1 & 12,5 & (disminución) & 10 \\
Tramo 2 & 15 & 0 & 5 \\
Tramo 3 & 17,5 & 16,7 & 5 \\
Tramo 4 & 20 & 33.3 & 5 \\
Tramo 5 & 22,5 & 50 & 20 \\
Tramo 6 & 25 & 66.7 & 40 \\
\hline
\end{tabular}

* Estimaciones de la Generalitat de Catalunya.

Fuente: Generalitat de Catalunya (2012).

En resumen, los principios de equidad no forman parte de la determinación de los importes de los precios públicos en el sistema catalán, sino que se aplican a posteriori a través del sistema complementario de becas. Las limitaciones de

${ }^{19}$ En base a las estimaciones de la Generalitat de Catalunya reproducidas en la Tabla 2, la aportación media de los estudiantes catalanes equivaldría al $18.4 \%$. En el SUE dicho valor estaría en un intervalo de entre el $25,25 \%$ y el 35,25\%, aplicando, respectivamente, el rango mínimo y máximo del R. D. En Italia ha sido del 12\% entre los años 2001 y 2009 (Verde y Fia, 2011). 
este sistema se refieren a que la minoración de los importes está condicionada a la disponibilidad presupuestaria (art. 1.3 y 4, AGAUR, 2012) y, por otra parte, a que la distribución de los diferentes tramos de renta familiar pone en duda la real efectividad en términos de equidad del sistema implementado, ya que su diferenciación resulta particularmente reducida. Además, los resultados alcanzados por el nuevo sistema en su primer año resultan aún lejos de las estimaciones iniciales y de representar una sólida alternativa, ya que, según los datos del AGAUR (2013a) y del MECD (2014), las becas Equitat han sido presentadas por el $13,14 \%$ de los estudiantes matriculados y se han otorgado sólo al $8,82 \%$ de estos últimos, los que suponen un $67,17 \%$ de otorgadas/presentadas en el curso pasado. A pesar de todo esto, en el sistema catalán destaca, respecto al resto de las CC. AA., la introducción de un mecanismo de diferenciación de los precios, que intenta subsanar la regresividad del sistema lineal de precios públicos establecido a nivel estatal.

\section{VALORACIÓN DE UN SISTEMA PROGRESIVO DE PRECIOS PÚBLICOS}

De los apartados anteriores se desprende que existen argumentos teóricos que dan soporte a un incremento en los precios públicos universitarios, siempre y cuando el importe de los precios no suponga una barrera de entrada en el caso de rentas bajas, esto es, no tenga implicaciones negativas en términos de equidad. El objetivo fundamental de este apartado es detenernos en cuáles serían las consecuencias de introducir progresividad en el cobro de los precios públicos, con el objetivo de evitar uno de los efectos menos favorables de dicho incremento. En primer lugar, sería necesario valorar lo que se pretende obtener en términos de recursos, tanto en cantidades como en su reparto. Así, sería necesario determinar si el incremento en el peso de la financiación que recae en el estudiante sirve para reducir el esfuerzo público (reducción presupuestaria de la financiación pública), o si este aumento se refleja en un crecimiento del gasto total en educación universitaria (sin reducción del gasto público). Si además la subida de precios se realiza de forma progresiva, es necesario determinar cómo se distribuye ese reparto entre los grupos de renta. Esto es, si se quieren obtener los mismos fondos pero conseguidos de manera progresiva (los importes pedidos a los escalones más altos de renta compensan los importes que se dejan de cobrar de los inferiores), o bien si se quiere elevar la aportación de todos los grupos de renta para aumentar la recaudación final. En el primer caso, los aumentos de los últimos grupos de renta no serían muy elevados. En el segundo, los colectivos de mayor renta podrían llegar a contribuir por el coste real del servicio educativo, aunque habría que establecer cuál sería el límite máximo para la fijación de estos precios, que podría establecerse según el precio de un grado similar en una universidad privada. O también cuál es el ahorro que le 
supone a un estudiante de renta alta el realizar estudios en una universidad pública, y que puede destinar a una serie de gastos complementarios en bienes y servicios educativos, como máster, prácticas no remuneradas, cursos de idiomas en el extranjero, etc.

Otra de las cuestiones fundamentales al plantear un sistema progresivo en precios se refiere a la fijación de los escalones de renta y a los importes cobrados en cada uno de ellos. Por una parte, la diferenciación por tramos de renta tiene que ser lo suficientemente fina como para que no haya grandes saltos que perjudiquen, fundamentalmente, a las clases medias-bajas, colectivo que no estará cubierto por becas o por exenciones de precios públicos y que puede ser más sensible a unos precios elevados. Por otra parte, sería necesario cuantificar bien el importe cobrado en cada tramo para que los efectos en la progresividad sean reales. Así, el caso italiano, progresivo a nivel teórico, no lo es tanto cuando se analiza en la práctica. Pacifico (2009) estudia la incidencia global del sistema de precios italiano y encuentra que el sistema de precios públicos no beneficia claramente a los grupos de renta baja, sino a las clases medias, que cuentan con una subvención elevada vía gasto público y unas tasas reducidas por no pertenecer a los tramos altos de renta. Esto se debe, por un lado, a que existe una parte de la contribución estudiantil fija (y que por tanto afecta más a las rentas bajas), por otro, a que las universidades no establecen unos precios lo suficientemente elevados para las rentas más altas que permitan alcanzar una progresividad real y, finalmente, a la dificultad de comprobación de la veracidad de los ingresos de los hogares por parte de las universidades italianas.

Otro de los aspectos fundamentales, y que ya se ha señalado con anterioridad, se refiere a la libertad con la que cuentan las universidades italianas, las cuales pueden establecer diferentes importes de contribución estudiantil. Esto implica una gran dispersión en los precios fijados, que pueden diferir enormemente entre universidades (por ejemplo, de los 290,80€ pagados en media en la universidad de Bari en el primer tramo de renta, a los 1.005,87€ pagados en Parma en la rama científica, también en el primer tramo de renta; de los $881,81 €$ pagados en el tramo de renta de los más ricos en la universidad de Salento, rama humanística, a los $3.500 €$ pagados en Pavía en la rama científica). Llevado al caso español, resulta recomendable tener en mente la experiencia italiana en términos de la libertad para la fijación de precios por parte de las universidades y sus implicaciones en términos de equidad, si se compara con el marco actual de enorme dispersión en el precio cobrado en las diferentes CC. AA. españolas (tal y como se señala en el trabajo de Escardíbul et al., 2014). Incidiendo en la cuestión de las cuantías de los precios públicos y su impacto en las rentas de los hogares, hay que señalar que la renta media por hogar español en 2010 fue de $25.094 €$ (INE, 2013), lo que supone que los precios públicos medios del curso $2012 / 13(1.074 €)$ supondrían un 4,2\% en el presupuesto anual de la media de 
los hogares ${ }^{20}$. Como es lógico, estos precios suponen un mayor esfuerzo a los hogares de menor renta $(13,4 \%$ y $9,6 \%$ para las decilas primera y segunda, respectivamente y un $2,9 \%$ y $2,2 \%$ para las dos últimas). En términos absolutos, un grado en la experimentalidad más cara para la media de las CC. AA. le cuesta 127,28€ mensuales (contando 10 meses de curso) en 2012/13 a un estudiante en primera matrícula, $76,39 €$ al mes en la más barata. En el caso de segundas y sucesivas matrículas, estas cantidades se verían incrementadas en un rango de $66,67 €$ a 106,32€ mensuales si estimamos un porcentaje de repetición del $25 \%$, de la experimentalidad más barata a la más cara. Si comparamos los valores medios con los costes asumidos por los hogares en otros niveles educativos, se comprueba que el gasto en este nivel no obligatorio no se aleja mucho del que realizan, por ejemplo, los hogares en educación infantil de primer ciclo (no obligatoria) en escuelas públicas, que asciende a los $752 €$ anuales (INE, 2012).

Otro de los temas relevantes se refiere a la potencial distribución de los precios en términos de progresividad y a su configuración regional: por una parte, no es deseable que se produzca una orientación del alumnado de menores recursos hacia los grados de más baratos, situación que podría tener una mayor relevancia ante aumentos de precios y un endurecimiento de los requisitos académicos de las becas (Moreno y Sánchez, 2003). Por otra, tampoco lo es que se establezcan diferencias regionales que afecten a la igualdad de oportunidades. En el caso italiano, se observa que la contribución media es más elevada para las universidades situadas en las regiones del norte (Federconsumatori, 2011), que son más ricas (ISTAT, 2013), como se aprecia en la Figura 3.

Además, estas universidades aplican un sistema de tasas y contribuciones más progresivo, ya que en media la diferencia entre el primer y el último tramo es de alrededor de $1.854 €$ frente los $994 €$ de las universidades del sur. Con ello, parecería existir cierta equidad inter-regional, puesto que los estudiantes cuyos hogares se sitúen en regiones más ricas, contribuirán en mayor medida al sufragio de la educación, puesto que su capacidad económica es superior y, además, la progresividad en su sistema de precios es mayor. Sin embargo, si este hecho no está correctamente recogido a través del sistema de precios (precios bajos para tramos de renta bajos) y de la política de becas, este esquema en realidad atentaría contra la igualdad de oportunidades, puesto que un estudiante procedente de una región con menos recursos tendría medios inferiores para poder acudir a una universidad situada en una región más cara, si ésta es su elección.

Al implementar un sistema progresivo también hay que tener en cuenta cuestiones burocráticas. Así, el sistema italiano cuenta con un formato de Declaración Sustitutiva Única (DSU), a través de la cual se certifica anualmente la

${ }^{20}$ Teniendo en cuenta que los datos relativos a ingresos en la Encuesta de Condiciones de Vida están infravalorados (como es común a este tipo de encuestas), si nos atenemos a los resultados de renta de Contabilidad Nacional, el peso de los precios académicos sobre la renta será menor. 
condición económica de los ciudadanos para solicitar cualquier prestación social, acudiendo a cualquier centro autorizado, y una vez que se obtiene, cada estudiante puede consultar el valor de lo que tendría que pagar en cada universidad en función de su renta. En España no existe este sistema unificado, pero sí que se aplica de forma similar en la petición de ciertos servicios públicos, como es la solicitud de una escuela infantil de primer ciclo (donde uno de los criterios que se valora es la menor renta del hogar), o en la de las becas generales en cualquier nivel educativo. Las nuevas tecnologías han facilitado enormemente la burocracia asociada a la declaración de los datos fiscales (y otros) para los ciudadanos, que únicamente tienen que autorizar la consulta de estos datos a las administraciones para verificar su situación económica.

Figura 3

Contribución estudiantil media (tasas y contribuciones universitarias) por tramos ISEE en Italia y por zonas geográficas (2011)

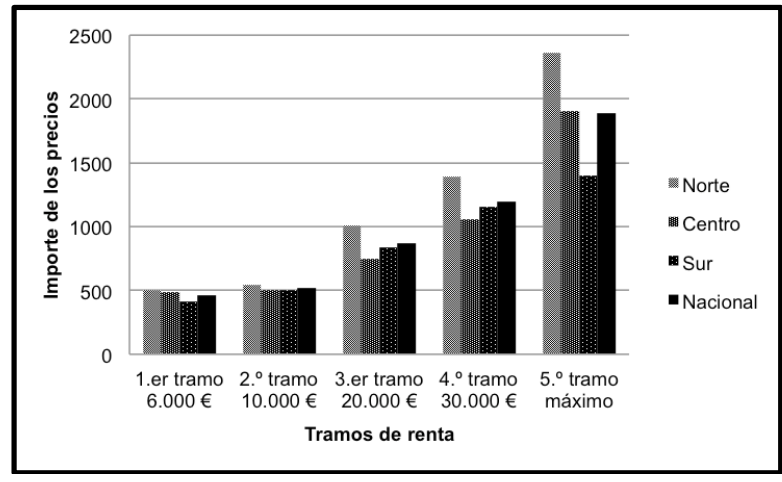

Fuente: Elaboración propia a partir de Federconsumatori (2011).

Por último, no podemos olvidar que un sistema que se basara en la certificación de las rentas de los hogares para cobrar precios tendría que tener en cuenta los problemas de fraude asociados a las concesiones de prestaciones públicas basadas en la declaración de ingresos (fundamentalmente, IRPF). Sin embargo, éste no es un problema atribuible a la parte de los gastos públicos, sino de los ingresos, y debería ser subsanado desde ese punto de vista.

\section{CONCLUSIONES}

En este trabajo se ha realizado un primer análisis de la viabilidad teórica y práctica de un sistema progresivo de precios públicos universitarios. A partir de un repaso de la teoría económica y de un análisis de la evolución reciente de nuestro sistema de financiación y del de países de nuestro entorno se han subrayado los puntos débiles de un sistema lineal en renta. En particular, para el caso 
español la situación económica presente de los hogares requiere de una articulación de mecanismos de igualdad de oportunidades efectivos al fijar los precios académicos, que eviten la falta de acceso o el abandono de una parte de los estudiantes, fundamentalmente con rentas medias-bajas. En el caso del sistema de becas, más que a las cuantías y a la cobertura de becarios (que no se han visto tan menoscabadas), parece que en estos momentos se requiere prestar especial atención a cuestiones críticas para los estudiantes como son la dilación en el cobro de las becas y las restricciones excesivas en los requisitos de acceso, que mejorarían enormemente el papel corrector de desigualdades de las becas.

Italia, y en menor medida Cataluña, presenta un sistema teóricamente idóneo para conciliar tanto los objetivos de equidad como los de suficiencia e inmediatez de recursos y compatible con el entorno socioeconómico español, a través de la articulación de una estructura progresiva de precios públicos universitarios. Sin embargo, precisamente las imperfecciones de estos sistemas, señaladas en este estudio, así como los aspectos prácticos descritos, permiten delinear los puntos clave a tener en cuenta en el diseño de una alternativa progresiva a la estructura lineal de precios públicos del SUE. Los resultados del análisis de las propiedades de progresividad en ambos territorios ponen de manifiesto que, si bien es factible y no muy costoso implementar un sistema progresivo en renta (bajo diferentes modalidades), su aplicación no garantiza la consecución inmediata de este resultado. Por otra parte, cabe subrayar que la introducción de un criterio de progresividad en la determinación de los precios públicos no constituye un elemento de duplicidad respecto a la progresividad del sistema tributario, sino que dicho principio -no exclusivo del sistema tributario- se aplica dentro de un esquema de financiación ya alternativo por sí mismo al sistema tradicional de financiación vía tasación, tal y como se ha mostrado en la segunda sección.

Finalmente, el análisis llevado a cabo en este estudio ha permitido sentar las bases para futuras investigaciones, que profundizarán el alcance redistributivo de un sistema progresivo de precios públicos y las condiciones necesarias para su implementación en todo el territorio español.

\section{REFERENCIAS BIBLIOGRÁFICAS}

AGÈNCIA DE GESTIÓ D'AJUTS UNIVERSITARIS I DE RECERCA (2012). Resolución ECO/1559/2012. Disponible en: http://www10.gencat.cat/agaur_web/generados/catala/ home/recurs/doc/equitat_dogc.pdf [Último acceso: 24 de mayo de 2013].

AGÈNCIA DE GESTIÓ D'AJUTS UNIVERSITARIS I DE RECERCA (2013a). Equitat 2012. Resum de la convocatòria. Disponible en: http://www10.gencat.cat/agaur_web/ generados/catala/home/recurs/doc/estadistiques_equitat_12.pdf. [Último acceso: 15 de abril de 2013]. 
AGĖNCIA DE GESTIÓ D'AJUTS UNIVERSITARIS I DE RECERCA (2013b). Resolución ECO/1577/2013. Disponible en: http://www10.gencat.net/agaur_web/generados/catala/ home/recurs/doc/bequi_2013_cat.pdf. [Último acceso: 15 de febrero de 2014].

ALBERT, C. Y ROIG, J.M. (2013) Tuition fees in Spanish Public Universities in the academic year 2012-13. IV Workshop on Economics of Education: Higher Education Financing Policy: Schemes and Effects. Barcelona. Disponible en http://www.ub.edu/ economiaempresa/butlleti/publicacio/wp-content/uploads/2013/06/ProgramWS2013_ EE.pdf5225bc48ac7e2.pdf [Último acceso: 19 de febrero de 2014].

BOLETÍN OFICIAL DEL ESTADO (2012a). Real Decreto-ley 14/2012. Disponible en: http://www.boe.es/boe/dias/2012/04/21/pdfs/BOE-A-2012-5337.pdf [Último acceso: 14 de enero de 2013].

BOLETÍN OFICIAL DEL ESTADO (2012b). Real Decreto 1000/2012. Disponible en: http://www.boe.es/boe/dias/2012/07/05/pdfs/BOE-A-2012-9007.pdf. [Último acceso: 28 de abril de 2013].

CONSEJO DE UNIVERSIDADES (2010). Documento de mejora y seguimiento de las políticas de Financiación de las Universidades para promover la excelencia académica e incrementar el impacto socioeconómico del Sistema Universitario Español (SUE). Disponible en: https://www.mecd.gob.es/dctm/ministerio/educacion/ universidades/educacion-superior-universitaria/finaciacion/2010-documento-financiacion.pdf? documentld=0901e72b800b7241. [Último acceso: 25 de noviembre de 2013].

DIARIO OFICIAL DE LA GENERALITAT DE CATALUNYA (2012). Decret 77/2012. Disponible en: http://portaldogc.gencat.cat/utilsEADOP/PDF/6169/1253048.pdf. [Último acceso: 28 de abril de 2013].

ESCARdíBul, O., PÉREZ-ESPARRELlS, C., MORALES, S., DE LA TORRE, E. (2014). Precios de matrícula en las enseñanzas universitarias por Comunidades Autónomas (1992-2013): un análisis antes y después del Espacio Europeo de Educación Superior. Comunicación presentada al XXI Encuentro de Economía Pública, Girona.

FEDERAZIONE NAZIONALE CONSUMATORI E UTENTI (2011). $2^{\circ}$ rapporto nazionale Federconsumatori sui costi degli atenei italiani. Disponible en: http://www.federconsumatorier.it/news/universita-il-rapporto-sugli-atenei-italiani. [Último acceso: 15 de octubre de 2011].

GARCÍA-PEÑALOSA, C., WÄLDE, K. (2000). Efficiency and equity effects of subsidies to higher education [versión electrónica]. Oxford Economic Papers, n $52,702-722$.

GAZZETTA UFFICIALE (1997). Decreto del Presidente della Repubblica del 25 luglio 1997, $\mathrm{n}^{\circ}$ 306. Disponible en: http://www.gazzettaufficiale.it/do/gazzetta/serie_generale/ 3/pdfPaginato?dataPubblicazioneGazzetta=19970916\&numeroGazzetta=216\&tipoSerie= SG\&tipoSupplemento=GU\&numeroSupplemento=0\&edizione=0\&elenco30giorni=\&numP agina=4. [Último acceso: 8 de agosto de 2013].

GAZZETTA UFFICIALE (1998). Decreto Legislativo del 31 marzo 1998, $\mathrm{n}^{\circ}$ 109. Disponible en: http://www.gazzettaufficiale.it/do/gazzetta/serie_generale/3/pdfPaginato? dataPubblicazioneGazzetta=19980418\&numeroGazzetta=90\&tipoSerie=SG\&tipoSupple mento=GU\&numeroSupplemento=0\&edizione=0\&elenco30giorni=\&numPagina=4. [Últim o acceso: 8 de agosto de 2013].

GENERALITAT DE CATALUNYA (2012). Nova política de preus públics i beques del sistema universitari català. Disponible en: http://premsa.gencat.cat/pres_fsvp/docs/ 2012/06/29/14/15/b08792ab-3392-4dcf-86d9-43b616f8af83.pdf [Último acceso: 23 de abril de 2013]. 
HERNÁNDEZ ARMENTEROS, J. Y PÉREZ GARCÍA, J.A. (2011). Nuevas perspectivas para la financiación y el gobierno de las Universidades. Studia XXI. Fundación Europea Sociedad y Educación, 6, Madrid.

INSTITUTO NACIONAL DE ESTADÍSTICA (2012). Encuesta sobre el Gasto de los Hogares en Educación. Curso 2011-2012.

INSTITUTO NACIONAL DE ESTADÍSTICA (2013). Encuesta de Condiciones de Vida, año 2011.

ISTITUTO NAZIONALE DI STATISTICA (2013). Noi Italia. Pil pro capite. Disponible en: http://noi-italia.istat.it/index.php?id=7\&user_100ind_pi1\%5Bid_pagina\%5D=490\& cHash=038652ddd44feef5b96b697c374bf31f [Último acceso: 8 de agosto de 2013].

MARTÍN VALLESPÍN, E. (2009). El papel de la financiación en el Proceso de Bolonia: un análisis de la suficiencia, eficiencia y equidad de los modelos de financiación universitaria en Europa. Presupuesto y Gasto Público, 55/2000, 121-139.

MINISTERIO DE EDUCACIÓN, CULTURA Y DEPORTE (2011). Estadísticas de precios públicos en enseñanzas universitarias. Series históricas.

MINISTERIO DE EDUCACIÓN, CULTURA Y DEPORTE (2013a). Datos y cifras del SUE. Curso 2012-2013.

MINISTERIO DE EDUCACIÓN, CULTURA Y DEPORTE (varios años). Estadísticas de precios públicos universitarios. Cursos 2008-2009 a 2013-2014.

MINISTERIO DE EDUCACIÓN, CULTURA Y DEPORTE (2014). Avance de la Estadística de estudiantes universitarios. Curso 2012-2013. Disponible en: $\quad$ http://www.mecd.gob.es/educacion-mecd/areaseducacion/universidades/estadisticas-informes/estadisticas/alumnado/2012-2013.html [Último acceso: 10 de febrero de 2014].

MINISTERIO DE HACIENDA Y ADMINISTRACIONES PÚBLICAS (VARIOS AÑOS). Presupuestos Generales del Estado, Tomo VIII, Sección 18, Ministerio de Educación, Programa 323M: Becas y Ayudas a Estudiantes.

MORENO HERRERO, D. Y SÁNCHEZ CAMPILLO, J. (2003). ¿Están cambiando las titulaciones que cursan los becarios? [versión electrónica] En Actas de Economía de la Educación AEDE XII, coord. por Rafael Zorrilla Torras, María Jesús San Segundo Gómez de Cadiñanos, 499-513. Oviedo, 18 y 19 septiembre de 2003.

OCDE (2008) Tertiary Education for the Knowledge Society: VOLUME 1 : Special features: Governance, Funding, Quality.

OCDE (2013). Education at Glance: OECD Indicators, OECD Publishing. Disponible en: http://dx.doi.org/10.1787/eag-2013-en [Último acceso: 15 de febrero de 2014].

PACIFICO, D. (2009). Inequality and higher education in Italy. The distributive impact of fees and subsidies to academics. CAPPapers, $\mathrm{n}^{\circ}$ 69, 1-22. Disponible en: http://www.capp.unimore.it/pubbl/cappapers/Capp_p69.pdf [Último acceso: 7 de julio de 2013].

SALAS VELASCO, M. (2008). Economía de la Educación. Madrid: Prentice-Hall.

SAN SEGUNDO GÓMEZ DE CADIÑANOS, M.J. (2005). Economía de la educación. Madrid: Síntesis.

VERDE, M., FIA, M. (2011). Le risorse finanziarie e cognitive del sistema universitario italiano. Uno sguardo d'insieme. POLIS Working Papers, 191. Disponible en: http://polis.unipmn.it/pubbl/RePEc/uca/ucapdv/verde191.pdf [Último acceso: 1 de junio de 2013]. 